\title{
Displasia grave de colo uterino em paciente idosa: relato de caso
}

\author{
Severe cervical dysplasia in an elderly patient: case report
}

Displasia cervical severa em mujeres de edad avanzada: reporte de caso

Tatiane Nicolela Prata Costa ${ }^{1 *}$, Márcia Cristina Taveira Pucci Green ${ }^{1}$, Giovana Ferreira de

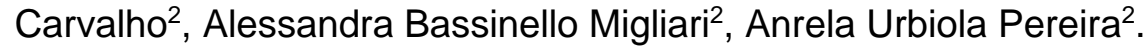

\section{RESUMO}

Objetivo: Relatar um caso de displasia grave de colo uterino em paciente idosa e alertar para a importância da realização dos exames de rastreamento além do preconizado pelo Ministério da Saúde, para prevenir câncer e realizar tratamento precoce de displasias. Detalhamento do caso: Aborda-se o caso de uma paciente de 80 anos, atendida no Ambulatório de Ginecologia do município de Franca, no estado de São Paulo, assintomática, aparentemente sem fatores de risco, que apresentou ao rastreamento de câncer de colo uterino uma neoplasia intraepitelial de alto grau. Foi, então, encaminhada para colposcopia, que confirmou o diagnóstico por meio do exame anatomopatológico. Considerações finais: Conclui-se que é muito importante o rastreamento do câncer de colo uterino além da idade preconizada pelo Ministério da Saúde, que atualmente é de 64 anos, tendo em vista o modo de evolução da doença, o aumento da expectativa de vida e as mudanças sociais atuais, com maior tempo de exposição aos fatores de risco.

Palavras-chave: Neoplasias do colo do útero, Idosa, Rastreamento.

\section{ABSTRACT}

Objective: The aim of the present paper is to report a case of severe cervical dysplasia in an elderly women and to warn the importance of carrying out screening tests even when patients are beyond the recommended by the Brazilian Ministry of Health, to prevent cancer and perform early dysplasia treatment. Case details: It is a case of an 80-years-old patient, treated at a Gynecology clinic, in Franca, in the state of São Paulo, asymptomatic, apparently without risk factors, presenting a high-grade intraepithelial neoplasia for cervical cancer screening. She was then referred for colposcopy, which confirmed the diagnosis through the anatomopathological examination. Final considerations: It has been concluded that the screening of cervical cancer is very important beyond the age of 64 recommended by the Ministry of Health in view of the disease evolution, the increase in life expectancy and the current social changes, with longer exposure to risk factors.

Keywords: Uterine cervical neoplasms, Aged, Diagnosis.

\section{RESUMEN}

Objetivo: Informar un caso de displasia cervical severa en una paciente anciana y advertir sobre la importancia de realizar pruebas de detección además de las recomendadas por el Ministerio de Salud, para prevenir el cáncer y realizar un tratamiento temprano de la displasia. Detalles del caso: Es un caso de una paciente de 80 años tratada en la Clínica de Ginecología para pacientes ambulatorios en el municipio de Franca, en el estado de São Paulo, asintomática, aparentemente sin factores de riesgo, que presentó una neoplasia intraepitelial de alto grado. Luego fue derivada para una colposcopia, que confirmó el diagnóstico mediante un examen anatomopatológico. Consideraciones finales: Se concluye que es muy importante detectar el cáncer de cuello uterino más allá de la edad recomendada por el Ministerio de Salud que actualmente es de 64 años, con base en la forma y tiempo de la evolución de la enfermedad, como el aumento de la esperanza de vida y los cambios sociales actuales, con mayor exposición a factores de riesgo.

Palabras clave: Neoplasias del cuello uterino, Anciano, Diagnóstico.

${ }^{1}$ Fundação Santa Casa de Misericórdia de Franca, Franca - SP. *E-mail: tati_npc@hotmail.com 2Universidade de Franca (UNIFRAN), Franca - SP. 


\section{INTRODUÇÃO}

O câncer de colo de útero (CCU) é a quarta maior causa de morte por câncer entre as mulheres no Brasil, ficando atrás de neoplasia de mama, pulmão e cólon e reto, segundo dados estatísticos do INCA de 2017, tornando-se, assim, um grande problema de saúde pública. Estima-se 16.590 novos casos de câncer do colo do útero em 2020, no Brasil, representando 7,4\% do total de neoplasias em mulheres (INCA, 2020).

O carcinoma de células escamosas é o tipo histológico mais comum de CCU, representando $70 \%$ dos casos (DIZ MDP e MEDEIROS R, 2009). As lesões precursoras para esse tipo de câncer são chamadas de neoplasias intraepiteliais cervicais (NIC) e são divididas em três graus de acordo com o acometimento da espessura do epitélio cervical por células atípicas.

Assim, NIC I seria displasia leve com a presença de células atípicas em terço inferior do epitélio escamoso, NIC II, displasia moderada com células atípicas ocupando os dois terços inferiores desse epitélio, e NIC III, displasia acentuada/grave ou carcinoma in situ com células atípicas ocupando mais de dois terços ou toda a espessura do epitélio escamoso (AIDÉ S, et al., 2009).

A progressão de uma lesão precursora para o carcinoma é de aproximadamente 20 anos (CARVALHO R, et al., 2006) e o surgimento de NIC III sem detecção prévia de lesões de baixo grau pode ocorrer (AIDÉ S, et al., 2009).

Em estágios iniciais, o CCU é frequentemente assintomático. Quando há sintomas, apresenta-se como sangramento vaginal, dispareunia e corrimento, o qual pode ser aquoso, mucoide ou purulento e fétido. Quando há dor pélvica ou lombar, com irradiação para os membros, pode indicar doença avançada. Em casos mais graves, pode ocorrer invasão do trato urinário e/ou gastrointestinal (DIZ MDP e MEDEIROS R, 2009).

O principal fator de risco para neoplasias intraepiteliais é a infecção pelo Papilomavírus Humano (HPV), transmitido sexualmente, sendo os subtipos 16 e 18 oncogênicos e responsáveis por $32 \%$ das infecções no mundo (SANJOSÉ S, et al., 2007).

Outros fatores associados ao CCU são: tabagismo, multiparidade, outras doenças sexualmente transmissíveis, múltiplos parceiros, imunossupressão e características do hospedeiro relacionadas à regulação de genes virais (AIDÉ S, et al., 2009; DIZ MDP e MEDEIROS R, 2009).

O pico de incidência do CCU está entre 50 e 60 anos, com as taxas de mortalidade aumentando com o avançar da idade em todo país (VILAÇA MN, et al., 2012), apresentando 6.385 óbitos em 2017, o que corresponde a 6,2\% do total de óbitos por câncer em mulheres (INCA, 2020).

O rastreamento de lesões precursoras de CCU é feito através do exame de Papanicolau (exame citopatológico do colo uterino), que possui alta especificidade (SASLOW D, et al., 2002; RICHARDSON LA, 2011) na detecção de células atípicas suspeitas. A realização adequada do exame de Papanicolau e do tratamento das lesões precursoras em mulheres entre 25 e 65 anos de idade pode reduzir a mortalidade pela doença em 80\% (DIZ MDP e MEDEIROS R, 2009).

O Ministério da Saúde preconiza o rastreamento do CCU dos 25 aos 64 anos, dando ênfase às mulheres em período fértil e com vida sexual ativa. Para mulheres com mais de 64 anos e que nunca realizaram o exame de Papanicolau, é preconizado realizar dois exames com intervalo de um a três anos. Caso os dois exames sejam negativos, essas pacientes podem ser dispensadas de exames adicionais (INCA, 2011).

No entanto, a expectativa de vida tem aumentado, assim como a exposição aos fatores de risco para CCU, e, por isso, é importante não descartar o rastreamento a partir dessa idade, já que podem ser encontradas lesões de alto grau que evoluíram ao longo dos anos e que não foram detectadas previamente (RIBEIRO JF, et al., 2016).

Dessa forma, esse estudo tem como objetivo relatar um caso de displasia grave de colo de útero em paciente idosa e alertar a importância da realização de exames de rastreamento acima de 64 anos, para prevenir $\mathrm{CCU}$ e realizar tratamento precoce de displasias, tendo em vista o aumento da expectativa de vida da população feminina somado ao maior tempo de exposição aos fatores de risco para o câncer de colo uterino. 


\section{DETALHAMENTO DO CASO}

Os dados colhidos foram do prontuário de uma paciente do sexo feminino, de 80 anos, atendida no Ambulatório de Ginecologia do município de Franca, no estado de São Paulo, tendo como morbidades hipertireoidismo e dislipidemia, em uso de Tapazol, Sinvastatina e Amitriptilina. Com história prévia de câncer de pele tratado cirurgicamente e história ginecológica de 5 gestações, 4 partos normais e 1 aborto, sendo viúva, há 40 anos sem relação sexual, menopausada há 25 anos. Realizou terapia hormonal, porém, não sabia informar em consulta tempo de uso e nome da medicação. Sem relato de tabagismo ou etilismo.

Em consulta de rotina com ginecologista, negando qualquer queixa, apresentou em exame citopatológico, colhido em fevereiro de 2017, atipia de células escamosas de significado indeterminado. Com esse resultado, a conduta foi realizar novo exame em 6 meses.

Em novembro de 2017, foi colhido exame citopatológico que demonstrou resultado de lesão intraepitelial de alto grau (NIC II/NIC III - HSIL) sendo, então, encaminhada para colposcopia, onde foi encontrado epitélio acetobranco leve ao exame com ácido acético e áreas de iodo negativo (às 3 horas e entre 10-11h). Foi realizada biópsia às $10-11 \mathrm{~h}$, com retirada de três fragmentos de tecido para análise. O resultado do exame microscópico da biópsia, de fevereiro de 2018, indicava Neoplasia intraepitelial cervical de alto grau - NIC II/NIC III. Foi solicitada conização para tratamento.

A conização foi realizada em junho de 2018 e o resultado do exame anatomopatológico, com fragmentos de lábio anterior e posterior de colo e de canal cervical, confirmou neoplasia intraepitelial de alto grau (NIC III) em lábio anterior e canal cervical. Desde então, a paciente acompanha semestralmente em ambulatório escola com realização de coleta de citopatológico e realização de colposcopia, até os dias de hoje, sem alterações.

\section{DISCUSSÃO}

Dentre os fatores de risco para CCU apresentados pela paciente, os de maior relevância seriam o fator idade e fatores relacionados ao próprio organismo da paciente. Também é importante lembrar sobre a possibilidade de relações sexuais extraconjugais ou pré-matrimoniais (até mesmo do companheiro), pensando no risco associado à infecção pelo vírus HPV ao apresentar contato com múltiplos parceiros. No entanto, no caso citado, não há história de múltiplos parceiros e nem de outros fatores de risco para CCU como tabagismo, multiparidade (acima de sete filhos o risco de neoplasia de colo aumenta quatro vezes em relação às nulíparas) ou uso de anticoncepcional (ANJOS SJSB, et al., 2010).

A história natural do CCU tem evolução lenta, de cerca de 15 a 20 anos, envolvendo uma série de etapas desde a infecção do colo uterino pelo vírus do HPV até o desenvolvimento do câncer invasivo. A evolução de lesões de baixo grau para as de alto grau acontece em cerca de $2 \%$ dos casos em 5 anos e destes, 2/3 evoluem para carcinoma em 20 anos (FRAZER I, 2007).

A lesão apresentada no caso pode ter iniciado como uma lesão intraepitelial de baixo grau e avançado gradualmente ou já surgido como de alto grau, e, como o desenvolvimento desse tipo de câncer é lento, a boa expectativa de vida da paciente contribuiria para o desenvolvimento de um carcinoma invasor caso a mesma não realizasse o exame de Papanicolau (AIDÉ S, et al., 2009).

$\mathrm{Na}$ fase pré-invasiva, o carcinoma invasor é quase sempre assintomático, corroborando com o relato (CARVALHO R, et al., 2006). O diagnóstico da doença foi através do exame citopatológico, de rastreamento, que apresentou alteração de significado indeterminado, necessitando ser repetido em 6 meses de acordo com o Ministério da Saúde e, com um segundo exame alterado, foi realizada colposcopia seguindo o mesmo protocolo (INCA, 2016). Tal fato nos atenta sobre a importância do rastreamento, pois a falta de sintomas não exclui a presença do câncer, mesmo em formas avançadas.

O rastreamento do CCU é feito pelo exame citopatológico e sua realização no Brasil é preconizada dos 25 aos 64 anos, sendo que até o ano de 2010 era previsto a abrangência do rastreio até os 59 anos apenas (RIBEIRO JF, et al., 2016). Isso devido ao pico de incidência ocorrer na faixa de 45 a 50 anos (IBGE, 2018; 
DIZ MDP e MEDEIROS R, 2009). Porém, torna-se relevante repensar sobre a importância do rastreio do CCU acima dos 64 anos, pois o aumento da idade se relaciona diretamente com o aumento do número de casos de neoplasias malignas e, no Brasil, o número de idosas tende a aumentar nos próximos anos (SANTOS MS, et al., 2011), sendo que a expectativa de vida da mulher brasileira no ano de 2017 foi de 79,6 anos (IBGE, 2018).

Em mulheres abaixo de 30 anos, a maioria das infecções por HPV regride espontaneamente; entretanto, acima dessa idade, tendem a ter menor taxa de regressão (THULER LCS, et al., 2012). Em um estudo realizado por Thuler em 2012, com 77.317 casos de câncer provenientes dos Registros Hospitalares do Câncer, a média de idade das mulheres ao diagnóstico de câncer do colo do útero foi de 49 anos, entretanto, observou-se $17 \%$ de mulheres com diagnóstico acima de 64 anos, indicando que uma em cada cinco mulheres diagnosticadas estava fora da faixa etária preconizada para o rastreio. Além disso, as taxas de câncer cervical parecem aumentar acentuadamente em vários países cerca de 10 anos após cessar a triagem (SASIENI P, et al., 2010).

Um estudo com 105 mulheres de 45 a 64 anos, acompanhadas por 7 anos, apresentou prevalência de HPV de 34\%. Dentre as participantes, 24\% apresentavam tipos oncogênicos de alto grau, sendo os tipos mais comuns o HPV-16 (72\%) e o HPV-31 (16\%), com uma taxa de persistência da infecção de 16\% no período do estudo. O que apoia a necessidade de triagem contínua em mulheres pós-menopausa para detectar lesões pré-neoplásicas (SMITH EM, et al., 2004).

O organismo envelhecido possui maior risco de desenvolver doenças crônico-degenerativas, como neoplasias relacionadas à sexualidade, as quais correspondem a causas expressivas de morbi-mortalidade na terceira idade (SANTOS RFA, et al., 2015). A expectativa de vida das mulheres é de 7 anos a mais que os homens (SANTOS MS, et al., 2011) e, 17\% delas, entre 75 e 85 anos, referem ser sexualmente ativas (FLEURY HJ e ABDO CH, 2015). Isso deixa muitas idosas vulneráveis a essas doenças e, associado à falta de informação, não induz a um comportamento de prevenção contra o CCU (SANTOS MS, et al., 2011).

No geral, as idosas realizam menos o exame citopatológico e estudos revelam que dentre os motivos estão a maior atenção dada às mulheres em período fértil, a própria cultura brasileira de negligenciar os exames de rastreio após a menopausa, a baixa escolaridade, o baixo nível socioeconômico (RIBEIRO JF, et al., 2016), medo, vergonha, desinformação (SANTOS MS, et al., 2011) e a falta de incentivo de práticas sexuais seguras, por ainda serem consideradas como indivíduos que pararam de manter relações sexuais (SANTOS RFA, et al., 2015).

As mulheres idosas possuem maior risco de adenocarcinoma invasivo devido à dificuldade de reconhecimento precoce das anomalias displásicas que podem aparecer nos exames. Além disso, devido ao hipoestrogenismo, a junção escamo-colunar (JEC) pode não ser visível, tornando exame colposcópico insatisfatório, o que dificulta o diagnóstico precoce de lesões (BRUNO MT, 2019).

Outro fator que corrobora com a importância do rastreamento do CCU em mulheres acima da idade preconizada pelo Ministério da Saúde é que esse câncer é mais invasivo nessas pacientes em comparação com mulheres mais novas (SANTOS RFA, et al., 2015), porém, a resposta ao tratamento é efetiva em todas as idades (RIBEIRO JF, et al., 2016).

Um fator importante a ser considerado em relação ao tratamento conservador da NIC II ou III é a recidiva. Cerca de $10 \%$ das mulheres submetidas à conização terá recidiva, sendo que esta não está relacionada com as margens do cone, uma vez que mais de $70 \%$ das mulheres com margens comprometidas ao serem submetidas à histerectomia não apresentaram doença residual e cones com margens livres podem ter recorrência por doença multifocal, inadequação da avaliação do material cirúrgico ou persistência do HPV (DERCHAIN SFM, et al., 2005).

Após o tratamento de NIC II e NIC III, a infecção pelo HPV geralmente é eliminada, e a persistência do vírus é o principal fator relacionado à recorrência da doença (DERCHAIN SFM, et al., 2005). Devido a isso, é importante manter acompanhamento das mulheres que tiveram diagnósticos e tratamentos, como foi realizado com a paciente em questão. 


\section{REFERÊNCIAS}

1. AIDÉ S, et al. Neoplasia intraepitelial cervical. DST - Jornal Brasileiro de Doenças Sexualmente Transmissíveis, 2009; 21(4): 166-170.

2. ANJOS SJSB, et al. Fatores de risco para câncer de colo do útero segundo resultados de IVA, citologia e cervicografia. Revista da Escola de Enfermagem da USP, 2010; 44(4): 912-20.

3. BRUNO MT, et al. Management of ASC-US/HPV positive post-menopausal woman. Virology Journal, 2019; 16(39).

4. CARVALHO R, et al. Carcinoma de células escamosas microinvasivo - Relato de caso. Revista Paranaense de Medicina, 2006; 20(3): 65-69.

5. DERCHAIN, SFM, et al. Neoplasia intra-epitelial cervical: diagnóstico e tratamento. Revista Brasileira de Ginecolia e Obstetrícia, 2005; 27(7): 425-433.

6. DIRETRIZES BRASILEIRAS PARA O RASTREAMENTO DO CÂNCER DO COLO DO ÚTERO / INSTITUTO NACIONAL DE CÂNCER JOSÉ DE ALENCAR GOMES DA SILVA. Coordenação de Prevenção e Vigilância. Divisão de Detecção Precoce e Apoio à Organização de Rede. - 2. ed. rev. atual. - Rio de Janeiro: INCA, 2016.

7. DIRETRIZZES BRASILEIRAS PARA O RASTREAMENTO DO CÂNCER DO COLO DO ÚTERO / INSTITUTO NACIONAL DE CÂNCER. Coordenação Geral de Ações Estratégicas. Divisão de Apoio à Rede de Atenção Oncológica. - Rio de Janeiro: INCA, 2011.

8. DIZ MDP, MEDEIROS R. Câncer de colo uterino - fatores de risco, prevenção, diagnóstico e tratamento. Revista de Medicina, 2009; 88(1): 7-15.

9. FLEURY HJ, ABDO CH. Sexualidade da mulher idosa. Revista Diagnóstico e Tratamento, 2015; 20(3): $117-20$.

10. FRAZER I. Correlating immunity with protection for HPV infection. International Journal of Infectious Diseases, 2007; 11(2): 10-16.

11. IBGE - Instituto Brasileiro de Geografia e Estatística. Tábua completa de mortalidade para o Brasil - 2017. Rio de Janeiro, 2018.

12. INCA - Instituto Nacional de Câncer / Ministério da Saúde. Controle do câncer do colo do útero: conceito e magnitude. Disponível em: http://www.inca.gov.br. Acesso em: 1 maio 2020.

13. RIBEIRO JF, et al. Aspectos sociodemografico e clínico da mulher idosa com câncer do colo do útero. Revista de Epidemiologia e Controle de Infecção, 2016; 6(2): 63-67.

14. RICHARDSON, LA. Optimizing technology for cervical cancer screening in high-resource settings. Expert Review of Obstetrics \& Gynecology. 2011; 6(3):343-353.

15. SANJOSÉ S, et al. Worldwide prevalence and genotype distribution of cervical human papillomavirus DNA in women with normal cytology: a meta-analysis. The Lancet infectious diseases, 2007; 7(7): 453-459.

16. SANTOS MS, et al. Saberes e praticas de mulheres idosas na prevenção do câncer cervico-uterino. Revista Brasileira de Enfermagem, 2011; 64(3): 465-471.

17. SANTOS RFA, et al. Conhecimento de idosas sobre o exame citopatológico. Revista de Enfermagem UFPE online, $2015 ; 9(2): 517-25$.

18. SASIENI P, et al. What is the right age for cervical cancer screening? Journal Women's Health. 2010; 6(1):1-4.

19. SASLOW D, et al. American Cancer Society Guideline for the Early Detection of Cervical Neoplasia and Cancer. CA: a Cancer Journal for Clinicians, 2002; 52 (6): 342-362.

20. SMITH EM, et al. Persistent HPV infection in postmenopausal age women. International Journal of Gynecology and Obstetrics, 2004; 87(2): 131-137

21. THULER LCS, et al. Perfil das Pacientes com Câncer do Colo do Útero no Brasil, 2000-2009: Estudo de Base Secundária. Revista Brasileira de Cancerologia, 2012; 58(3): 351-357.

22. VILAÇA MN, et al. Diferenças nos Padrões de Tratamento e nas Características Epidemiológicas entre Pacientes Idosas e Adultas Portadores de Câncer do Colo do Útero. Revista Brasileira de Cancerologia 2012; 58(3): 497-505. 